Special issue of the International Conference on Computational and Experimental Science and Engineering (ICCESEN 2014)

\title{
Developing Hardware and Software Appropriate to Phototrichogram Method
}

\author{
İ.S. ÜNCÜ ${ }^{a, *}$ AND O. KÖSE ${ }^{b}$ \\ ${ }^{a}$ Süleyman Demirel University, Technology Faculty, \\ Electrical and Electronic Engineering Department, Isparta, Turkey \\ ${ }^{b}$ Süleyman Demirel University, Graduate School of Applied and Natural Sciences, \\ Electronic and Computer Education Department, Isparta, Turkey
}

\begin{abstract}
Visual method of hair analysis in medicine is called phototrichogrametry. Method is based on the interpretation of two image showing the physical changes of the hair. The photos are taken in green light, exposing both thin and thick hairs. By the help of the software the density of facial hair is determined and intensity of growth is assessed. Length increment can be approximately determined also. standardization, objectivity, reliability, decreasing errors and saving time make this method valuable healing experiment platform.
\end{abstract}

DOI: 10.12693/APhysPolA.128.B-384

PACS: 87.57.-s, 87.57.Ra

\section{Introduction}

Phototrichogram (FTG) helps to appoint the grow time of the beard in a short time [1]. In FTG photos of area which was shaved about half centimetre are taken. The ratio of anagen/telogen is determined from other digital photos from of same area [2-4]. Information provided by these photos allows to estimate the ratio of the growth of the hair and amount of the growing hair. This analysis is fulfilled by the background difference algorithm. The aim of this work is to acquire the essential image in more accurate, objective, easy to apply and clear way. In daily life some errors are made, like taking images of different areas each time. In specific situations this case can be evaluated with the Wilcoxon signal test [2]. In short, this process gives opportunity to interpret the objects as two distinctive groups and avoids the measuring of different hairs.

The observations of white or thin hair with FTG are difficult [3]. To make calculations easier the contrast degree of the image is increased. This gives more accurate results [5]. In the study some anagen and telogen hair were identified $[6,7]$. FTG gives the opportunity of measuring the speed of hair growth, and its density. For linear growth, by using millimetres scale, the software prepared according to reference points comments. The importance of this study is to decrease the other applicable methods. The necessity of dying of thin and light hair and initial shaving of the measurement area are the disadvantages of this method [8-11]. In FTG, anagen and telogen hair can be determined. It gives opportunity to measure the density, growth of the hair $[3,6]$.

This analysis is fulfilled by the background difference algorithm. This work advantage is that this work

*corresponding author; e-mail: serkanuncu@sdu.edu.tr provides observation of the changes by the help of previous digital photos.

The aim of this work is to acquire the essential image more accurate, objective, easy to apply and clear way.

In the study existence of some anagen and telogen hair was confirmed [6,7]. FTG indicates to give the opportunity of the speed of hair growth and density measurements. For linear growth the millimetre reference system is prepared by the software according to the set reference points.

The important point of this study is reducing the requirements of other applicable methods. The necessity of dying of thin and light hair and shaving the area are the disadvantages of this method [8-11]. FTG, as it is already said, anagen and telogen hair can be determined. It gives opportunity to measure the density and growth speed of the hair $[3,6]$.

\section{Material and method}

$\mathrm{C} \#$ and SQL programming languages were used to obtain phototrichogram. SQL provides keep the records and reach the data again. $\mathrm{C} \#$ provides keeping of examined individual and repositioning him again, analyse of the images, determination of number of hairs in chosen area and registering the results of the graphical analysis. Analytical software is designed for interaction with the user. It becomes possible with the help of DLL definitions and the prepared software. The image taken from the digital camera gives the opportunity to interpretation. The interpretation of those images is composed to get approximate results. The analysis cap is designed to get necessary image. In this study two cameras linked to step motor take the images. With the step motor controlled by an analysis software camera is positioned according to different coordinate data. The mechanical design and the size of the motor and reductors is adapted to proximity of a human head. The adjustment ability of this 


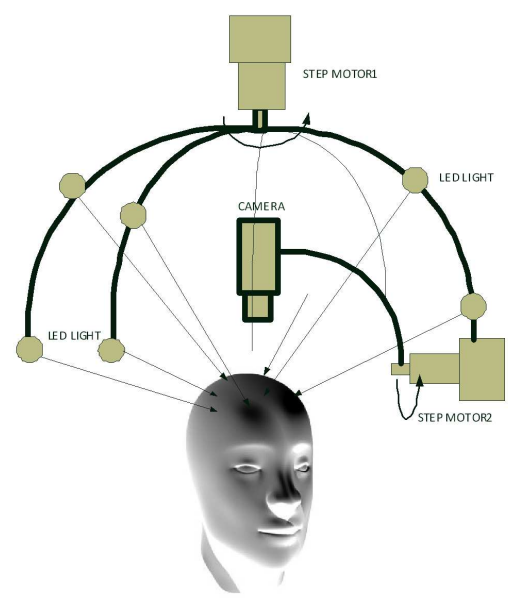

Fig. 1. The design of the analysis pot.

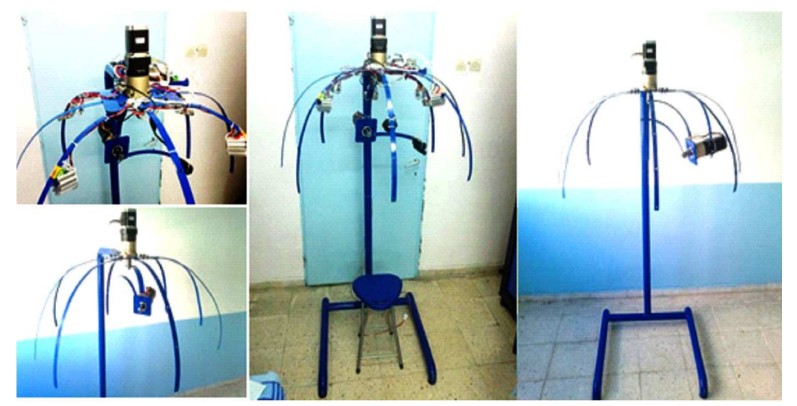

Fig. 2. The body of the analysis pot.

contraption allows it to move in $20 \mathrm{~cm}$ radius. The system moves circularly and with this movement the camera can be sited wherever wanted. The hardware photosystem is shown in Figs. 1 and 2.

The white light makes beard shining. When the green light is applied the beard turns black and hairy skin. Whether the hair body is thick or not under the green light it becomes black. Obtained images are shown in Fig. 3.
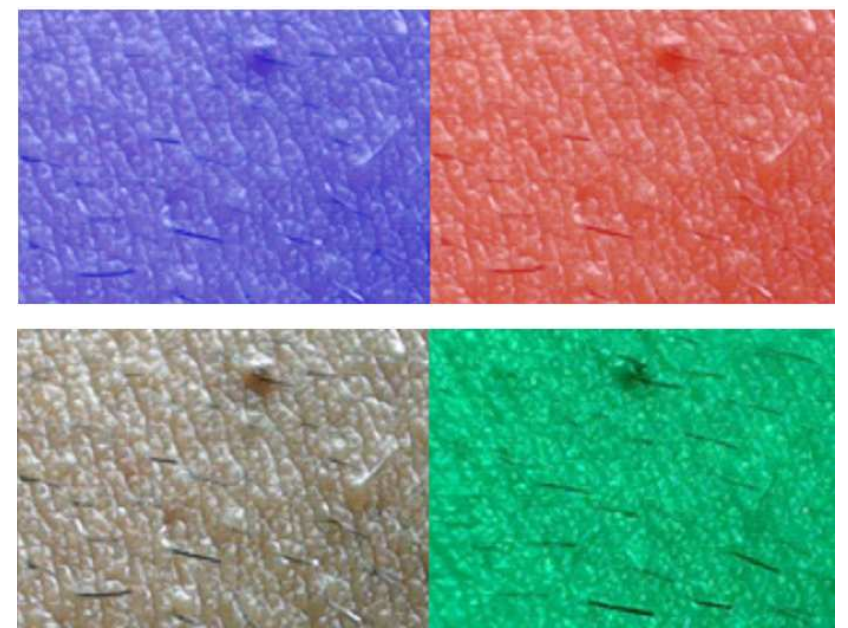

Fig. 3. The images of the hairy skin taken in different coloured light.
To avoid the shining spots histogram equalization is used. This process is effectuated on the pixels of the image according to the Eq. (3.1)

$$
f(a)=\sum_{r=1}^{a} \frac{n_{1}(r)}{N} .
$$

Here; $n_{1}(r)$ is density function, $r$ is the number of choosen pixels, and $N$ is total number of pixels.

\subsection{Application interface form}

With the help of analysis pot and software image can be taken by digital camera. On the basis of these images the growth of the hair, the individual type of hair cure or hair transplantation and the hairy area healing and closing density take place can be determined. The results determined from the taken images are recorded in the database.

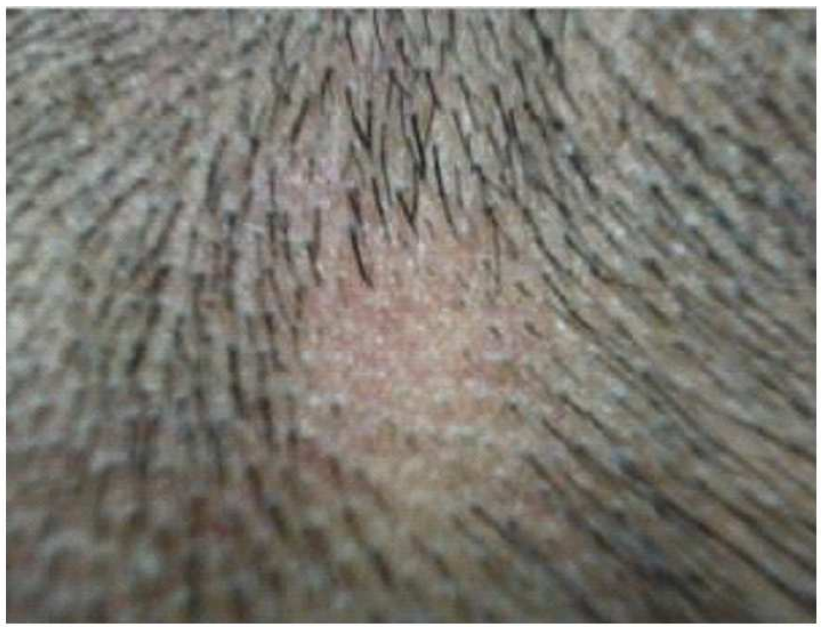

Fig. 4. Choosing of the analysis area.

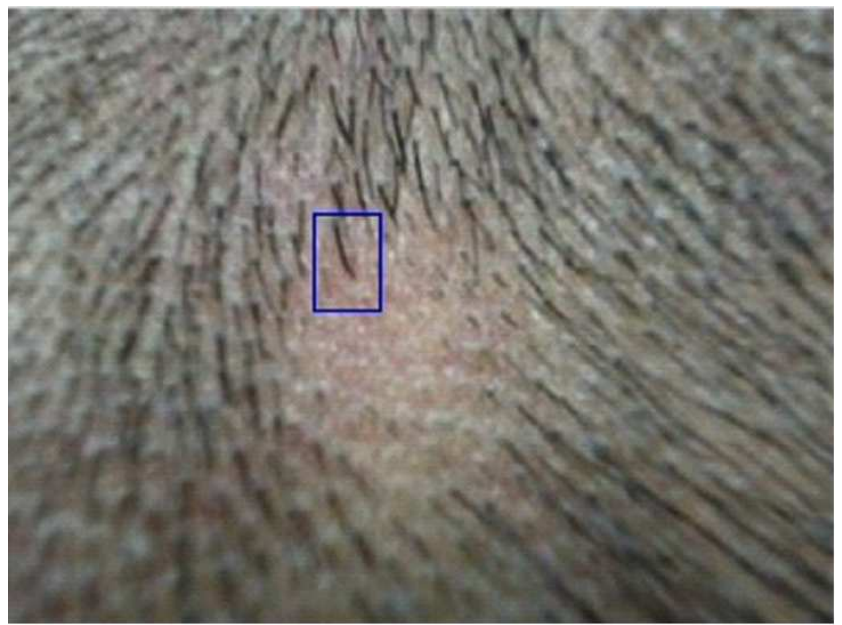

Fig. 5. Choosing of the hair for the analyse.

Before analyzing the image there is selection step. Photographed area is restricted to the desired coordinates as shown in Fig. 4. The sample hair which will be chosen from the area shown in Fig. 5. This process can be done 
under white or green light. Using different light sources is reasonable. This reason is to reduce distortion caused by the background effect to the method. Reducing the error quantity between the head skin and the beard gives the study the forward step. Interpretation of the images is fulfilled by taken attention to the pixel differences [4]. Image correction, filtering, partition, processes are also fulfilled. The importance of perfect lightening, image resolution and image identifying appears in section [12]. The images taken with digital camera proves the significance of the image size [13]. To delete parasites on the image we can get ideal value. The sample group is created upon the images taken according to the coordinates. This group forms from three object. But at the choosing step one should be careful about the clarity.

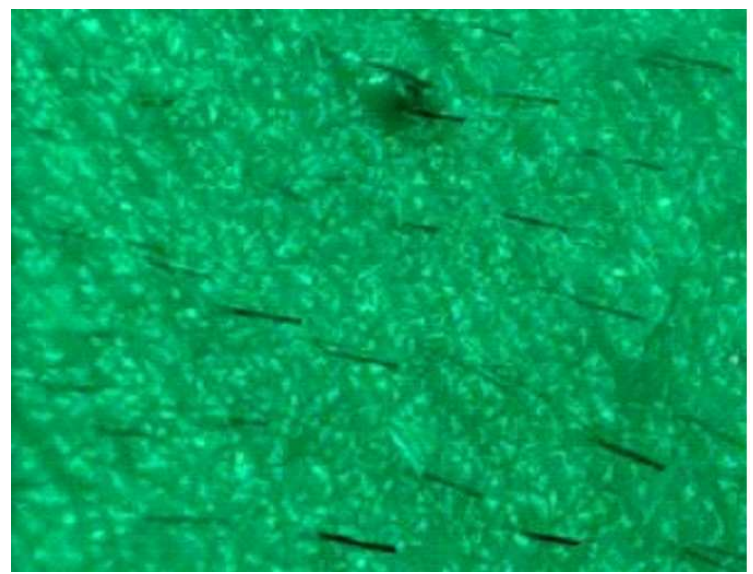

Fig. 6. The initial image of the hairy area.

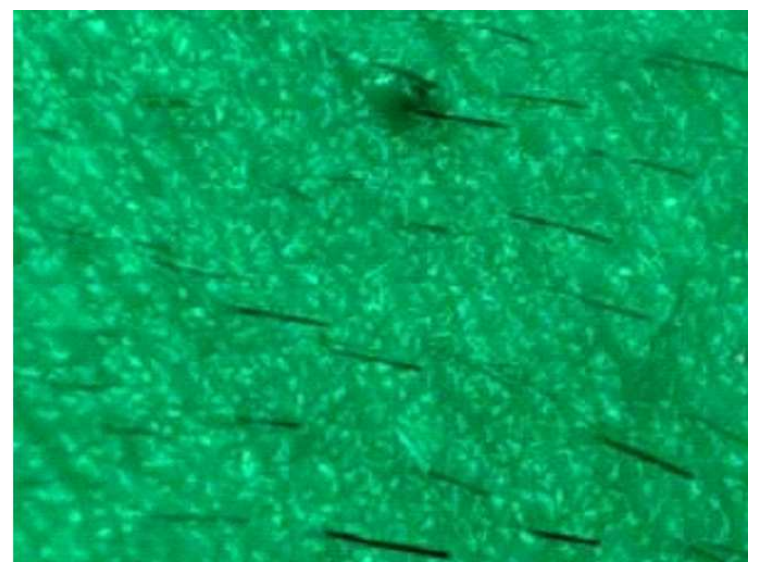

Fig. 7. The image of the hairy area after 72 hours.

In Figs. 6 and 7 some of the hairs are looking flu. Its choice as an option can hinder the true result. So the clear object should be chosen. Three selected hairs is enough for creation the sample group. This number can be increased or decreased. In the chosen sample group, as it shown in Figs. 8 and 9, the white framed hair grew and in the red framed area is supposed as not growing.

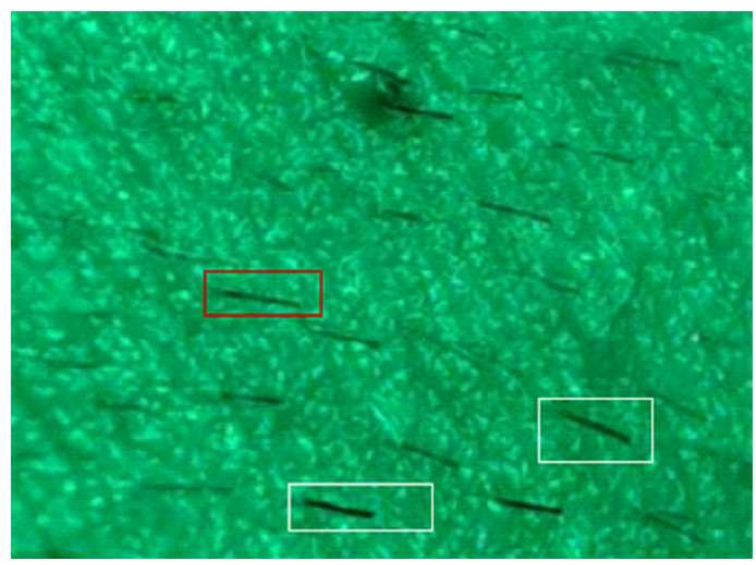

Fig. 8. Choosing sample from first image.

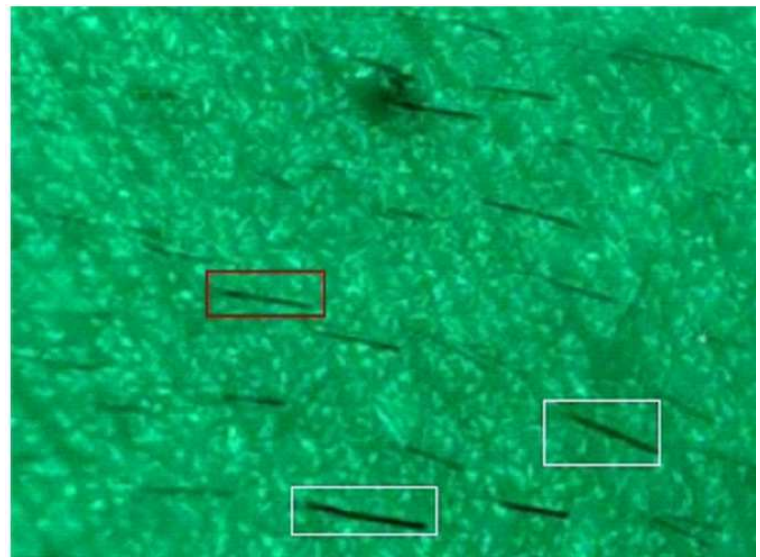

Fig. 9. Choosing sample from last image.

In this marking the limit value $0.7 \mathrm{~mm}$ is taken as basic [8]. It is assumed that the result under $0.7 \mathrm{~mm}$ means "not grown", the value above $0.7 \mathrm{~mm}$ is "grown". The exemplary screenshot illustrating the analysis of three hairs and numerical values are shown in Fig. 10.

\section{Conclusion}

In the analytical programme both images of the hairy skin can be seen. Hair chosen for measurements can be selected. Short shaved hairy area is needed as mentioned in literature. For long hair the results of method of image processing techniques are uncertain due to disturbances in the background difference method. Correct results are inaccessible in that case.

The same selection of hair is to be registered in both cases for this study. If the individual is sitting in a different position relative to the analysis cap the resultscan be also affected. Therefore taking the second image when the first image is not recorded in the computer for comparison may cause an error. Two different areas can not be compared each other. Measuring conditions should be the same as far as possible. 


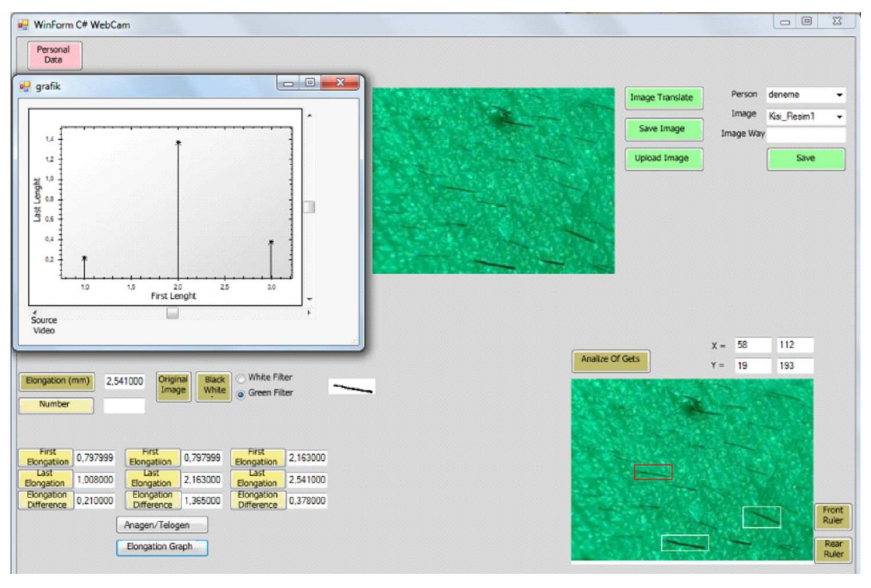

Fig. 10. The screenshot with the results of FTG analysis.

\section{Acknowledgments}

This work was supported in part by Scientific Research Projects Unit of Süleyman Demirel University (Project Number: 3448-YL1-13)

\section{References}

[1] A. Köşlü, Galenos 3, 29 (1999).

[2] D. Çetinkünar, M. Önder, Turkderm-Arch. Turk. D 46, 78 (2012).

[3] D. D'Amico, M. Vaccaro, F. Guarneri, F. Borgia, S. Cannavo, B. Guarneri, Eur. J. Dermatol. 11, 17 (2001).

[4] A. Yllmaz, M.Sc. Thesis, Golden Horn University of Science and Technology, Istanbul 2007.

[5] D.J.J.V. Neste, Eur. J. Dermatol. 11, 326 (2001).

[6] A.J. Chamberlain, R.P.R. Dawber, Austral. J. Dermatol. 44, 10 (2003).

[7] R. Paus, G. Cotsarelis, N. Engl. J. Med. 341, 491 (1999).

[8] R. Hoffmann, Eur. J. Dermatol. 11, 362 (2001).

[9] R. Hoffmann, J. Investig. Dermatol. Symp. Proc 8, 109 (2003).

[10] R. Hoffmann, Dermatology 211, 54 (2005).

[11] R. Hoffmann, Eur. J. Dermatol. 18, 65 (2008).

[12] H. Bal, M.Sc. Thesis, Gazi University, Ankara 2006.

[13] K. Asmaz, M.Sc. Thesis, Yildiz Technical University, Istanbul 2006. 